\title{
Research on Automobile Distance Measurement System on Highway Based on Vehicle-mounted Camera
}

\author{
Lu Zhiying, Han Chunyan \\ Department of Electrical Engineering and Automation \\ Tianjin University \\ Tianjin,China,300072 \\ Luzy@tju.edu.cn
}

\author{
Lin Lichen \\ Department of Electrical Engineering and Automation \\ Tianjin University \\ Tianjin,China,300072
}

\begin{abstract}
Poor visibility brings the safety issues to the automobile running on highway in fog weather. The research of vehicle distance measurement system can help driver better estimate the distance between the car and the car in front and make opportune anti-collision action even in poor visibility. Firstly, the related intrinsic parameters are obtained through camera calibration based on checkerboard image; Secondly, the geometric information of road markings and the position relationship between the car and the car in front are extracted from the images obtained by camera; Finally, the real distance is obtained by the transforms from image coordinate to camera coordinate and then to world coordinate with the camera intrinsic parameters. It is proved that the experimental result is similar to the real value and meets the requirement. Furthermore, the camera self-calibration method which can reduce the impact of human action and make the foundation for the further study on camera calibration, is proposed.
\end{abstract}

Keywords-Camera calibration, Corner extraction, Line detection, Distance measurement, Matlab simulation

\section{INTRODUCTION}

With the development of technology, the vehiclemounted camera has been improved greatly in film quality, size, appearance and cost saving. Now it has been applied to car accessories, such as electronic dog, vehicle reversing cameras.

The camera is installed in front of the car, so the vehiclemounted camera can get the same scene environment as the driver's view, capturing video image sequence files. These files reflect the real scene and fog information including the cars and all kinds of identifications on the roads. The research of recognition of highway landscape in fog weather based on video images is the precondition and significant base for building high quality, high performance scene window.

This work is focused on vehicle-mounted camera calibration which is a part of "Scenery recognition of highway in fog weather". The function of the camera calibration is to establish, the relationship between 2D Image coordinate and 3D Space coordinate, and then the distance between autos on highway can be obtained with the camera parameters and highway scene image information.

\section{The CAMERA CALIBRATION BASED ON CHECKERBOARD IMAGE}

\section{A. Camera calibration}

Camera calibration [1] is a classic problem in computer vision area and can establish relationship between 2D Image coordinate and 3D space coordinate. In the 3D reconstruction of the physical world, the CCD (Charge Coupled Device) camera is a basic measurement tool. The 2D Image of three-dimensional object obtained by CCD is in pixels. The relationship between three-dimensional geometry of a point in space object surface and its corresponding point in the image is determined by geometric model of camera image. The geometric model parameters are the camera parameters.

Camera parameters include intrinsic parameters and external parameters. The intrinsic parameters reflect optical and geometric properties of camera, such as: principal point, actual focal length, lens distortion and system calculation errors parameters. The external parameters reflect the position and orientation of the camera coordinate system relative to the world coordinate, such as rotation and translation parameters [1] [2].

Existing camera calibration techniques can be summarized into two categories: the traditional camera calibration method and camera self-calibration method. Linear calibration is commonly used in traditional camera calibration technique. However, due to the lack of the consideration of the nonlinear factors of camera lens, the accuracy of the results cannot meet the requirements of this project. In addition, the results obtained by various methods of camera self calibration are more sensitive to noise, poor in robustness, or have strict limitations on the movement of camera or target. Therefore, the traditional method of camera calibration based on standard reference (checkerboard image) was used in this subject.

\section{B. Camera calibration based on checkerboard image}

The method of camera calibration based on checkerboard can obtain camera parameters by using checkerboard image as a calibration reference. Therefore, digital image processing technique was needed in processing input image (checkerboard image) which includes (1) extracting the checkerboard image area from background (2) detecting the checkerboard region contour automatically (3) marking 
internal corners of the checkerboard image automatically and providing data for the camera calibration. The process of the camera calibration based on checkerboard image is shown in Figure1.

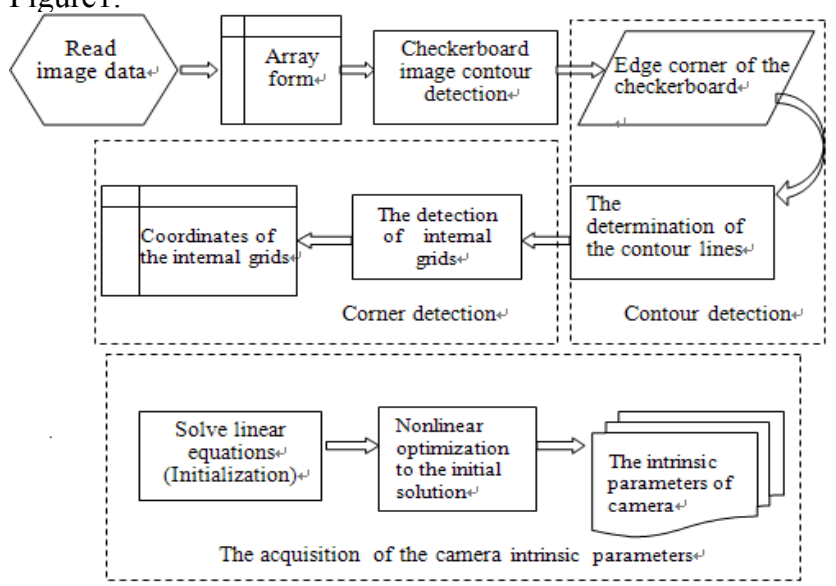

Figure 1. Flowchart of vehicle-mounted camera auto-calibration

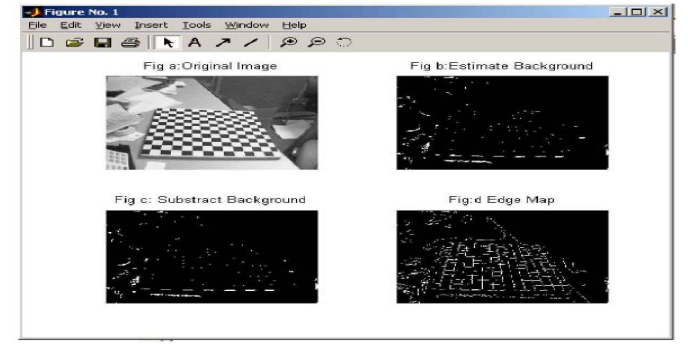

Figure 2. Results of background elimination and edge detection

1) Background elimination based on open operation

The main steps of background elimination: step1 Read image data, step2 Estimate background by opening operation in morphology, step3 subtract the background from original image, step4 Enhance image contrast.

In Figure2, Fig $\mathrm{a}$ is the original image, Fig $\mathrm{b}$ is the result of background estimation of the image, Fig $\mathrm{c}$ is the image without background.

2)The determination of checkerboard image contour lines

Corner detection: According to the characteristics of checkerboard image, the method of corner detection based on gray [3] is used in this paper.

The edge detection includes removing noise, differential operation, binarization and thinning in which differential operation is the key. According to the effect and speed of edge detection and the characteristics of research object, Prewitt operator [4] was used in this paper. Test result is shown in Fig d of Figure2, which will be used as the input of radon transform for line detection.

Radon transform [5], is often used for line detection. It can be interpreted as the projection of the image in space A. Every point in space A can be mapped to a straight line in space B. Because Radon transform is an integral of image pixels on each straight line, the high gray level lines in image form bright spots in space A, while the low gray value lines form dark spots in space A. So the detection of lines in image can be treated as the detection of bright and dark spots in space A.

In the left image of Figure3, the extreme point in bright regions corresponds to a straight line in the original image. The reconstructed image through Radon inverse transform is shown in the right image.

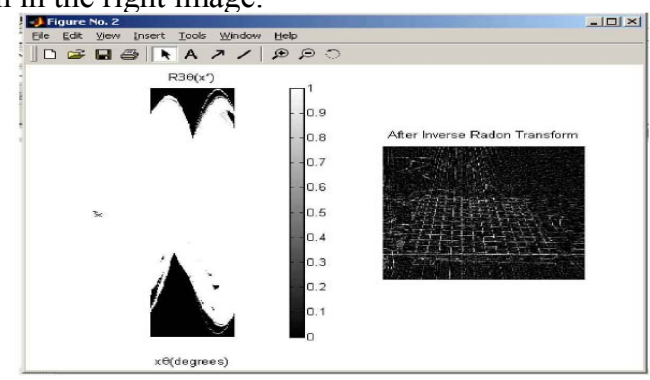

Figure 3. Result of Radon transform and inverse transform

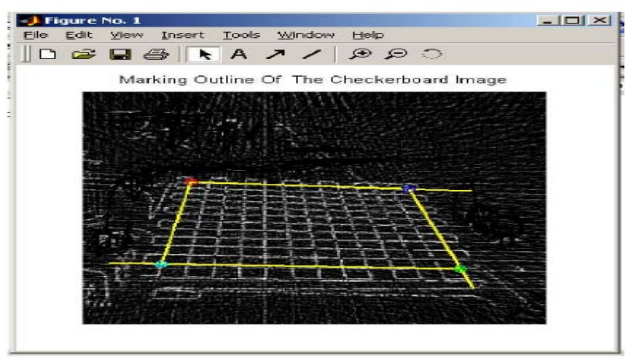

Figure 4. Result of contour lines detection

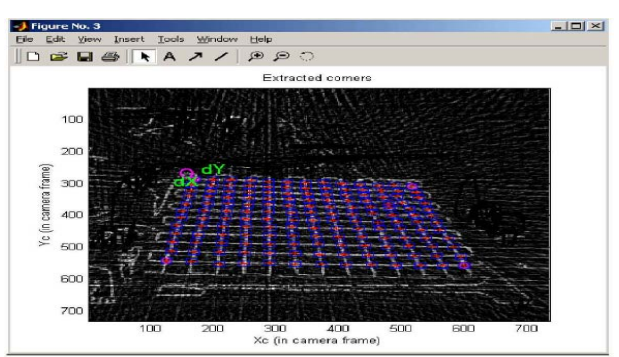

Figure 5. Result of internal grids detection

The steps of contour detection algorithm are shown as follows:

Step1: Read image data and do binarization; Step2: Calculate image size and estimate the regions in which exist contour corner point; Step3: Search the boundary point from the top left area through method of corner detection based on gray; Step4: Search edge lines in the checkerboard image through Radon transform and inverse transform; Step5: Make a straight line parallel to the chess grid through the boundary point; Step6: Search the boundary point in the top right area with contour corner; Step7: The point in straight line is approximated as the boundary point of the top right contour; Step8: Follow the steps 7,8,9, the bottom right and left boundary points can be found too; Step9: link the four contour points to obtain the outline of the checkerboard image. The result is shown in Figure4.

3)The Detection of internal grids of checkerboard image 
In this work, Harris operator [6] was used to detect internal grids. The algorithm steps were as following: From horizontal and vertical directions, the difference was detected between each image array and its adjacent elements circularly. If the difference were greater than 0 , the pixel was a corner point, otherwise the pixel was not a corner point. The result of corner detection is shown in Figure5. It can be seen, the feature points extracted by Harris operator are accurate, reasonable and better in distribution.

4)Camera calibration process

The intrinsic parameters of vehicle-mounted camera which is similar to ordinary camera in imaging principle reflect imaging model, so pinhole camera model was chosen as camera model. According to this model, the relationship between pixel coordinate and the normalized image coordinate expressed by intrinsic parameters of camera is shown in formula (1).

$$
\left[\begin{array}{c}
x_{p} \\
y_{p} \\
1
\end{array}\right]=K K\left[\begin{array}{c}
x_{d}(1) \\
x_{d}(2) \\
1
\end{array}\right]
$$
(2):

$\mathrm{KK}$ is the camera parameters matrix, defined in formula

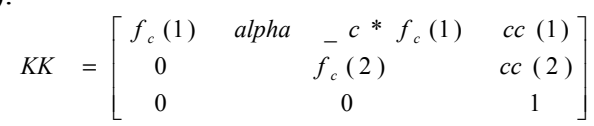

In $(1-1),[x p: y p]$ is pixel coordinate vector. $x d$ is normalized (pinhole camera model) coordinate vector of the image projection.

The main calibration process included two steps: the first step was the initialization of the equation. Without consideration of any case of lens distortion, initialization process was to solve linear equations and calculate the closed solution of the calibration parameters according to the data obtained by corner detection; the second step was nonlinear optimization of the initial solution. Nonlinear optimization method which is iterative method could minimize the projection errors of all calibration parameters in the least square sense.

The data obtained by image corner detection could be used to obtain intrinsic parameters of camera by solving equation. At least 5 images were needed to obtain intrinsic parameters of camera. The actual size of each checkerboard used in the experiment was known as $30 \mathrm{~mm} * 30 \mathrm{~mm}$, as shown in Figure6 and, the intrinsic parameters of camera obtained are shown in table 1.

\begin{tabular}{|c|c|c|c|}
\hline Parameter Name & Value & $\begin{array}{c}\text { Parameter } \\
\text { Name }\end{array}$ & Value \\
\hline Focal Length(fc) & $\begin{array}{l}{[658.0201} \\
2 ; 658.66 . \\
55]\end{array}$ & $\begin{array}{c}\text { Focal Length } \\
\text { Error(fc_erro } \\
\text { r) }\end{array}$ & $\begin{array}{l}{[1.70567 ;} \\
1.23334]\end{array}$ \\
\hline Principal point(cc) & $\begin{array}{l}{[303.1695} \\
6 ; 248.17 \\
626]\end{array}$ & $\begin{array}{c}\text { Principal } \\
\text { pointError(cc } \\
\text { error) }\end{array}$ & $\begin{array}{l}{\left[\begin{array}{l}1.72082 ; \\
2.61990]\end{array}\right.}\end{array}$ \\
\hline Skew(alpha_c) & $\begin{array}{l}{[0.00000} \\
]\end{array}$ & $\begin{array}{c}\text { Skew } \\
\text { Error(alpha_- } \\
\text { c error) }\end{array}$ & $\begin{array}{l}{[1.72082 ;} \\
2.61990]\end{array}$ \\
\hline
\end{tabular}

TABLE I. THE INTRINSIC PARAMETERS OF CAMERA

\begin{tabular}{|l|l|c|l|}
\hline Distortion(kc) & {$[0.25853 ;$} & Distortion & {$[0.00719 ;$} \\
& $0.14578 ; 0$ & Error(kc_err & $0.03428 ; 0$ \\
& $.00087 ;-$ & or) & $.00078 ;$ \\
& $0.00017 ; 0$ & & $0.00039 ;$ \\
& $.00000]$ & & $0.00000]$ \\
\hline Pixel error (er) & {$[0.13361 ;$} & & \\
& $0.12031]$ & & \\
\hline
\end{tabular}

\section{Highway Driving Distance MEasurement SYSTEM}

The key step was the method of vehicle-mounted camera calibration in the system. Firstly, the camera intrinsic parameters which reflect imaging model were obtained through vehicle-mounted camera calibration. Then, the geometric information of the road markings on the highway was obtained by processing and analysis to highway scene image. Finally, the corresponding relationship between 2D image coordinate and three-dimensional scene was obtained and the measurement of the distance between autos could be finished. The system chart is shown in Figure7.

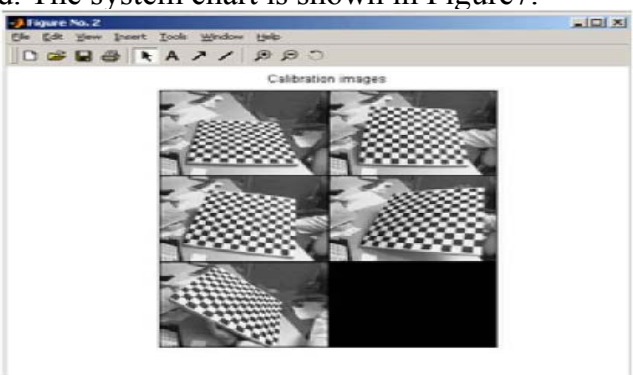

Figure 6. Schematic diagram of the input image

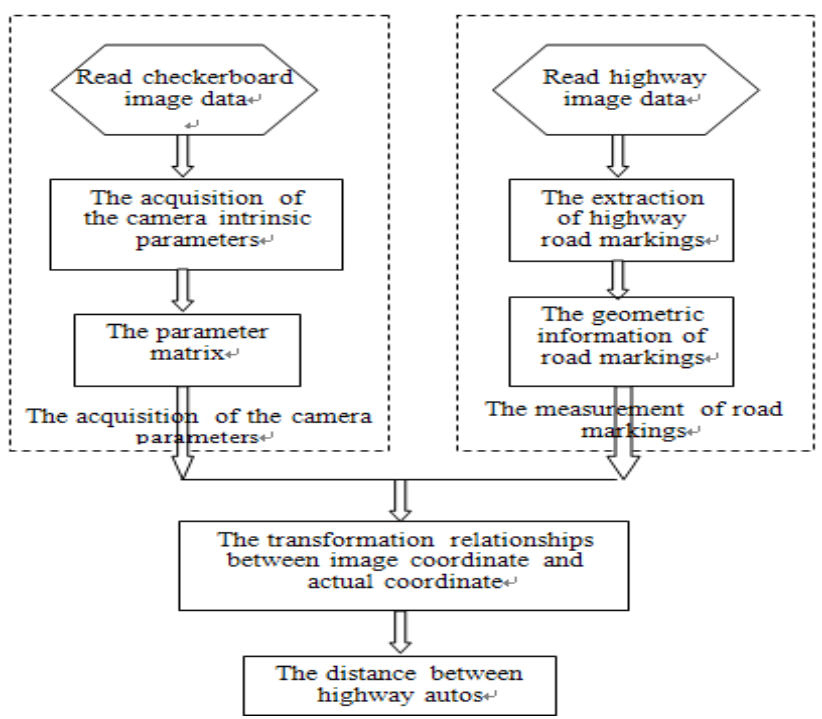

Figure 7. Highway driving distance measurement system chart

\section{A. Measurement of highway road markings}

Because the image information obtained by camera was based on the highway scene, so a description of the scene was needed. The following assumptions on highway model 
were made in this paper. (1) The width of highway was consistent and slow in change; (2) The direction of road changed slowly; (3) Changes in gray on the road were more stable(except for road markings);(4) The gray difference between road and the background was large ;(5) Road could not be very short.

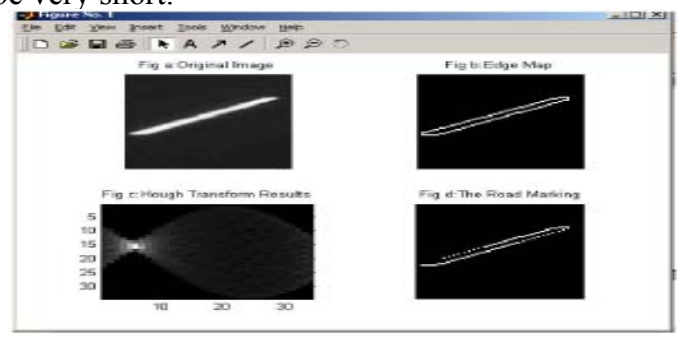

Figure 8. Line detection results based on Hough transform

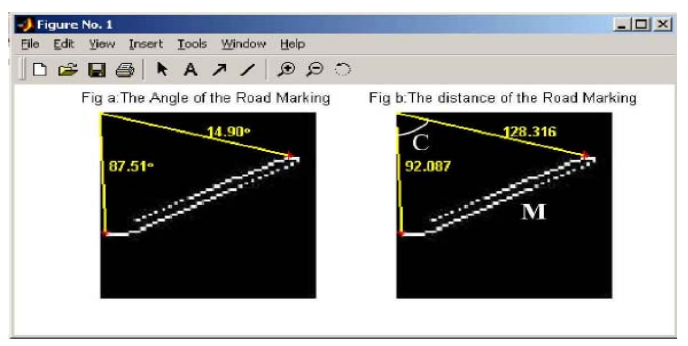

Figure 9. Geometric information of road markings

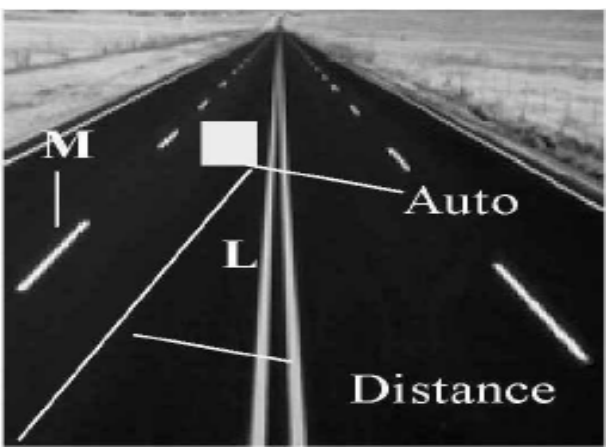

Figure 10. Highway scene image

According to the above assumptions, Hough transform was used to extract road markings. The compositions of the highway road are shown in Fig a of Figure8, and the corresponding result of Hough transform is shown in Fig d of Figure8. After finding the endpoints of the detected line, the module and angular of the endpoint vector in image coordinate were obtained as shown in Fig a of Figure9. The angle between the road tag line and horizontal direction was obtained through solving triangle as shown in Fig b of Figure9.

\section{B. System implementation}

Highway driving distance measuring system was divided into two steps:

(1)In accordance with the intrinsic parameters of camera, the geometric information of road markings were converted into length in camera coordinate system. The scale factor between real length and image length could be obtained by comparing the length in camera coordinate with the real length.

(2)According to the assemble position and field of view of the vehicle-mounted camera, the edge of the image was considered as the starting point of the line connecting the car with the car in front. According to the location of the front car, and in accordance with the same angle with the lane marker, the distance could be obtained in image coordinate, and converted into the real distance by the scale factor obtained in step (1).

According to the results of camera calibration and geometric information of road markings (Figure9), the conversion from image coordinate to camera coordinate and then to world coordinate could be done as follows:

Firstly, the conversion from image coordinate to camera coordinate was calculated. The two endpoints of road marker $M(4,92),(124,33)$ in image coordinate were substituted in formula (1-1):

$$
\begin{aligned}
& {\left[\begin{array}{c}
4 \\
92 \\
1
\end{array}\right]=\left[\begin{array}{ccc}
658.0195 & 0 & 303.1686 \\
0 & 658.6603 & 248.1772 \\
0 & 0 & 1
\end{array}\right]\left[\begin{array}{c}
x_{d}(1) \\
x_{d}(2) \\
1
\end{array}\right] \Rightarrow x_{d M 1}=\left[\begin{array}{l}
-0.4547 \\
-0.2371
\end{array}\right]} \\
& {\left[\begin{array}{c}
124 \\
33 \\
1
\end{array}\right]=\left[\begin{array}{ccc}
658.0195 & 0 & 303.1686 \\
0 & 658.6603 & 248.1772 \\
0 & 0 & 1
\end{array}\right]\left[\begin{array}{c}
x_{d}(1) \\
x_{d}(2) \\
1
\end{array}\right] \Rightarrow x_{d M 2}=\left[\begin{array}{l}
-0.2723 \\
-0.3267
\end{array}\right]} \\
& \text { dis }_{M}=\left|x_{d M 2}-x_{d M 1}\right|=0.2032
\end{aligned}
$$

The actual size of road marker (Rdis) was assumed as $1 \mathrm{~m}$, then, the scale factor $\mathrm{K}$ of road marker could be obtained as follows:

$$
K=\frac{\text { Rdis }_{M}}{\text { dis }_{M}}=\frac{1000}{0.2032}=4921.2598
$$

In step (2), the image used to get highway driving distance is shown in Figure10.The rectangle is the car tag through image segmentation. $\mathrm{L}$ is the distance required to obtain. To reduce computation, the dip of $\mathrm{L}$ is considered the same with the dip of M. The driving distance can be obtained by multiplying the image size of $\mathrm{L}$ with the scale factor $\mathrm{K}$.

The calculation method of $\mathrm{L}$ was same with the calculation method of $\mathrm{M}$ as mentioned in step (1). The length of $\mathrm{L}$ is:

$$
\operatorname{dis}_{D}=\left|x_{d d_{1}}-x_{d d_{2}}\right|=0.7769
$$

Then, the distance is $\mathrm{K} \times$ dis $\mathrm{D}=4921.2598 \times 0.7769=$ $3823.2 \mathrm{~mm}$, the distance between the car and the front car is $3.8 \mathrm{~m}$ as shown in Figure10.

\section{SUMMARIES}

In this paper, the automatic corner detection algorithm has been introduced before camera calibration, which could reduce system requirements for human intervention, and improve the speed of the camera calibration. In the scene extraction of highway, according to the characters of the target, the road marker of the highway was obtained based on Hough transform. The geometric information of the target object was obtained based on the geometric relationship of the target object in image coordinate. Finally, the results of the intrinsic parameters of camera were used in the conversion of the coordinates. The distance between vehicles was obtained based on the relationship between the road 
marker of the highway and the relative real distance in the real scene.

\section{REFERENCES}

[1] NIE Kai; LIU Wen-yao; WANG Jin-jiang. Camera Calibration Method with a Coplanar Target and Three-Dimensional Reconstruction, Proc. SPIE,[C], 2009, 7513, 75132W.

[2] WU Wen-qi; SUN Zeng-qi. Overview of Camera Calibration Methods for Machine Vision. Application Research of Computers [J],2004 PP:4-5.
[3] YIN Run-min; CHAI Xu-dong; LI Bo-hu. Corner Detection Algorithm Based on Gray Level Difference Analysis. Computer Engineering. [J], 2006 PP: 32-22.

[4] FAN Chun-ling; REN Yuan-yuan. Study on the Edge Detection Algorithms of Road Image. ISIP, [C],2010, PP:217-220.

[5] ZHANG Yu-hua; WANG Xin. Study of Finite Radon Transform in Face Recognition. ICCMS,[C],2010,PP: 22-24.

[6] XIE Dong-hai; ZHAN Zong-qian; JIANG Wan-shou. Improving Harris Corner Detector. Wtusm Bulletin of Science and Technology,[J],2003, PP:22-23. 